\title{
Rapamycin-Induced Abundance Changes in the Proteome of Budding Yeast
}

\author{
Chun-Shik Shin ${ }^{1}$, Yeon-Ji Chang ${ }^{1}$, Hun-Goo \\ Lee $^{2}$ and Won-Ki Huh ${ }^{1 *}$
}

${ }^{1}$ School of Biological Sciences, and Research Center for Functional Cellulomics, Institute of Microbiology, Seoul National University, Seoul 151-747, Korea, ${ }^{2}$ Department of Molecular Biology and Biochemistry, Rutgers University, Piscataway, New Jersey 08854, USA

\begin{abstract}
The target of rapamycin (TOR) signaling pathway conserved from yeast to human plays critical roles in regulation of eukaryotic cell growth. It has been shown that TOR pathway is involved in several cellular processes, including ribosome biogenesis, nutrient response, autophagy and aging. However, due to the functional diversity of TOR pathway, we do not know yet some key effectors of the pathway. To find unknown effectors of TOR signaling pathway, we took advantage of a green fluorescent protein (GFP)-tagged collection of budding yeast Saccharomyces cerevisiae. We analyzed protein abundance changes by measuring the GFP fluorescence intensity of 4156 GFP-tagged yeast strains under inhibition of TOR pathway. Our proteomic analysis argues that 83 proteins are decreased whereas 32 proteins are increased by treatment of rapamycin, a specific inhibitor of TOR complex 1 (TORC1). We found that, among the 115 proteins that show significant changes in protein abundance under rapamycin treatment, 37 proteins also show expression changes in the mRNA levels by more than 2-fold under the same condition. We suggest that the 115 proteins indentified in this study may be directly or indirectly involved in TOR signaling and can serve as candidates for further investigation of the effectors of TOR pathway.
\end{abstract}

Keywords: green fluorescent protein, proteome, rapamycin, Saccharomyces cerevisiae, TOR signaling pathway

\section{Introduction}

The target of rapamycin (TOR) kinase is highly con-

*Corresponding author: E-mail wkh@snu.ac.kr

Tel +82-2-880-9243, Fax +82-2-873-4740

Accepted 3 December 2009 served from yeast to human and specifically inhibited by a macrolide antibiotic, rapamycin. It is known that TOR kinase functions as a key regulator of eukaryotic cell growth and development (Wullschleger et al., 2006). TOR kinase exists in two functionally distinct protein complexes, TOR complex1 (TORC1) and TOR complex 2 (TORC2) (Loewith et al., 2002). Especially, rapamycin-sensitive TORC1 pathway plays critical roles in cell growth. TORC1 affects ribosome biogenesis by regulating transcription of all three kinds of RNA polymerases (Mayer and Grummt, 2006). Inactivation of TORC1 results in several physiological characteristics of nutrient starvation, including induction of autophagy (Rohde et al., 2001). Recently, it has been highlighted that TOR signaling is deeply involved in eukaryotic cell aging and aging-related diseases. Inhibition of TOR signaling extends lifespan in yeast, nematode and fruitfly (Jia et al., 2004; Powers et al., 2006; Zid et al., 2009). Although the knowledge about TOR pathway has been greatly expanded by intensive research over the last two decades, there are still many unknown factors in the upstream and downstream of TOR signaling pathway due to its complexity and crosstalk with other signaling pathways.

According to the central dogma of molecular biology suggested about 50 years ago (Crick, 1958), most of the genetic information in DNA is finally transferred to proteins. Proteins, the final products in the central dogma, are not only fundamental components of living cells but also mediators of most of the cellular processes. Thus, to broaden our understanding of cellular functions and processes, it is essential to know the mechanisms how to regulate the activity or abundance of each protein. On these days, rapid developing technology in life science makes it possible to study proteins at a large-scale or proteomic level. Proteomics has become well established as a term for studying proteins at a large scale. Therefore, proteomic research might be very helpful in understanding the precise cellular functions and processes.

In the present study, to gain a novel insight into TOR signaling pathway in Saccharomyces cerevisiae, we sought to analyze proteomic expression changes in yeast cells treated with or without rapamycin. To determine proteomic expression changes in yeast, we took advantage of a collection of 4159 green fluorescent protein (GFP)-tagged yeast strains (Huh et al., 2003). Previously, several researches have used the yeast GFP-tagged collection for rapid and precise high-throughput 
measurement of protein abundances which could be represented by GFP intensities tagged on each protein (Lee et al., 2007; Newman et al., 2006; Song et al., 2009). From our proteomic analysis, we show that 83 proteins are decreased whereas 32 proteins are increased by rapamycin treatment. We suggest that these 115 proteins may be directly or indirectly involved in TOR signaling and can serve as candidates for further investigation of the effectors of TOR pathway. Further study with these candidates may reveal important insights into TOR signaling pathway.

\section{Methods}

\section{Yeast strains, medium and reagents}

4159 GFP-tagged yeast strains expressing GFP fusion proteins (Huh et al., 2003) were used to monitor changes in protein levels. A synthetic complete medium (SC; $0.67 \%$ yeast nitrogen base without amino acids, $2 \%$ glucose) were prepared as previously described (Sherman, 2002). Cells were grown to the mid-log phase in SC medium at $30^{\circ} \mathrm{C}$. Rapamycin (Tecoland) was dissolved in DMSO and used at $200 \mathrm{ng} / \mathrm{ml}$ concentration.

\section{Quantitative proteomic analysis using a multi- label microplate reader}

GFP-tagged yeast strains were grown to the mid-log phase in 96 deep-well plates (Bioneer). The optical density (OD) and fluorescence intensity of the cells were measured in clear-bottom, 96-well plates (Greiner BioOne) with a multilabel microplate reader (EnVision; PerkinElmer). The OD was measured using a 595-nm filter. Fluorescence intensity was measured using an excitation filter of $488 \mathrm{~nm}$ and an emission filter of $535 \mathrm{~nm}$.

\section{GFP-intensity data processing and analysis}

To eliminate cellular autofluorescence, we set running median through OD with 3 parameters (window size: 0.01 OD; slide size: 0.002 OD; count threshold: 50 ). A relative abundance of each protein was calculated as:

$$
\begin{array}{r}
\text { Relative abundance of protein }=\text { fluorescence of } \\
\text { protein }^{\mathrm{OD}}-\text { running median }
\end{array}
$$

where fluorescence of protein $^{\mathrm{OD}}$ is a GFP intensity of each strain showing a certain $\mathrm{OD}$ and running median ${ }^{\mathrm{OD}}$ is an average GFP intensity of all strains showing the corresponding $\mathrm{OD}$. To exclude an experimental variation causing plate tendency, standard deviations of non-tagged cells and 44 plates containing 4159 GFP-tagged cells between GFP intensity measurements at 0,1 and $2 \mathrm{hr}$ were calculated, and 14 plates showing higher standard deviations than those of half of non-tagged cells were excluded for further analysis. Among the remaining proteins, we selected 115 proteins showing higher relative abundance values than $1.65 \times$ (standard deviations of non-tagged cells).

\section{Results}

Measuring protein abundance changes induced by rapamycin using a multilabel microplate reader

Physiological functions of a protein can be regulated at the level of abundance. Protein abundance changes result from a number of regulatory mechanisms ranging from transcription to post-translational modification. Thus, change in protein abundance can suggest a possibility of alteration in physiological roles of the protein. As a key regulatory signaling pathway of eukaryotic cell growth (Wullschleger et al., 2006), TOR pathway may be involved in the regulation of protein abundances. Based on this consideration, we hypothesized that protein abundance changes induced by rapamycin, a specific inhibitor of TORC1, might indicate involvement of the protein in TOR pathway. To test this hypothesis, we carried out a quantitative proteomic analysis with or without rapamycin treatment.

For a quantification of the yeast proteome, we took advantage of a collection of 4159 GFP-tagged yeast strains (Huh et al., 2003). Recently, several studies have shown that the yeast GFP-tagged collection can be used for profiling proteome expression by measuring the fluorescence intensity of each GFP-tagged strain (Lee et al., 2007; Newman et al., 2006; Song et al., 2009). To perform a proteomic quantification of the fluorescence intensities of the GFP-tagged yeast collection rapidly and precisely, we used a multilabel microplate reader that could simultaneously analyze the intensities of 96 GFP-tagged yeast cells. The validity of GFP-intensity data from a multilabel microplate reader was confirmed in our previous study (Song et al., 2009).

We measured the fluorescence of 4159 GFP-tagged yeast strains grown to the mid log-phase with or without treatment of $200 \mathrm{ng} / \mathrm{ml}$ rapamycin for $1 \mathrm{hr}$. During the data processing, we found that about $40 \%$ of raw data were not appropriate for further analysis and decided to exclude these data. The remaining 2422 GFPintensity changes under rapamycin treatment were further analyzed. Consequently, we found that 83 proteins were significantly decreased by rapamycin treatment whereas 32 proteins were increased under the same condition (Fig. 1). These 115 proteins that underwent 
significant protein abundance changes under rapamycin treatment are listed in Table 1.

\section{Comparison of protein abundance changes with mRNA level changes}

In the previous study, DNA microarray was used to examine the genome-wide transcription alteration under rapamycin treatment (Hardwick et al., 1999). Since a change in protein level usually originates from an alteration in the gene expression, we tried to compare the protein abundance changes with the mRNA level changes under rapamycin treatment. Among the 115 proteins that showed significant protein abundance changes, 37 proteins also showed $\geq 2$-fold changes in the mRNA level under rapamycin treatment. We investigated the correlation between the mRNA and protein level changes for these 37 proteins (Fig. 2). The Pearson correla-

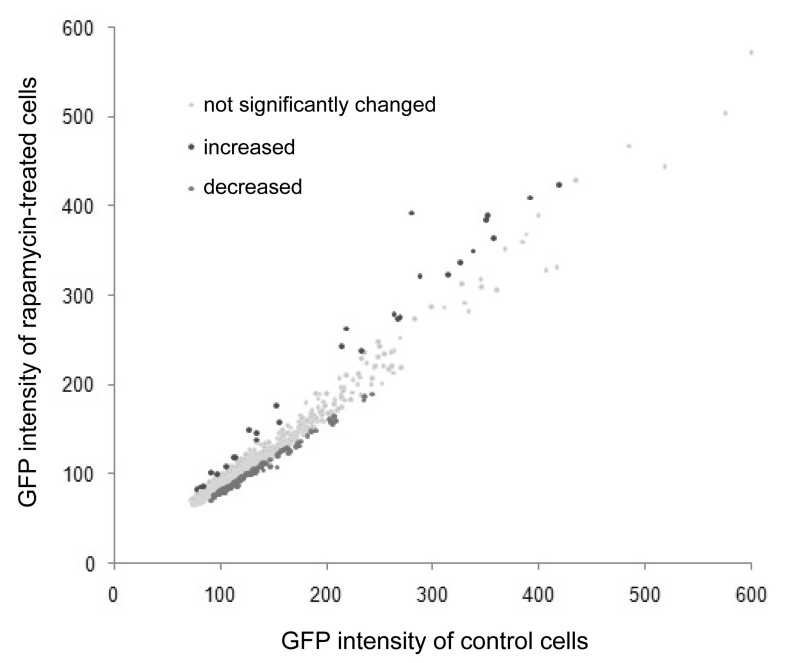

Fig. 1. Quantitative analysis of rapamycin-induced abundance changes in the yeast proteome. 4159 GFP-tagged strains were grown to the mid-log phase with or without treatment of $200 \mathrm{ng} / \mathrm{ml}$ rapamycin for $1 \mathrm{hr}$. GFP intensity values were normalized and plotted in log scale. tion analysis of 37 proteins showed a positive correlation between the mRNA and protein expression except for 3 proteins, Rck2, Eno1 and Mak10. These 3 proteins showed a negative correlation, and exhibited an increase in protein abundance while their mRNA levels were decreased under rapamycin treatment.

\section{Functional classification of 37 proteins showing changes in the protein and mRNA level by rapa- mycin}

The 37 proteins showing significant changes in the protein and mRNA level under rapamycin treatment were classified into the Gene Ontology (GO) categories (Ashburner et al., 2000). As shown in Table 2, the 37 proteins were enriched especially in the GO categories related with protein synthesis. This enrichment resulted from inclusion of 16 ribosomal proteins. It has been shown that TOR signaling pathway controls translation initiation by regulating elF2 $\alpha$ (Cherkasova and Hinne-

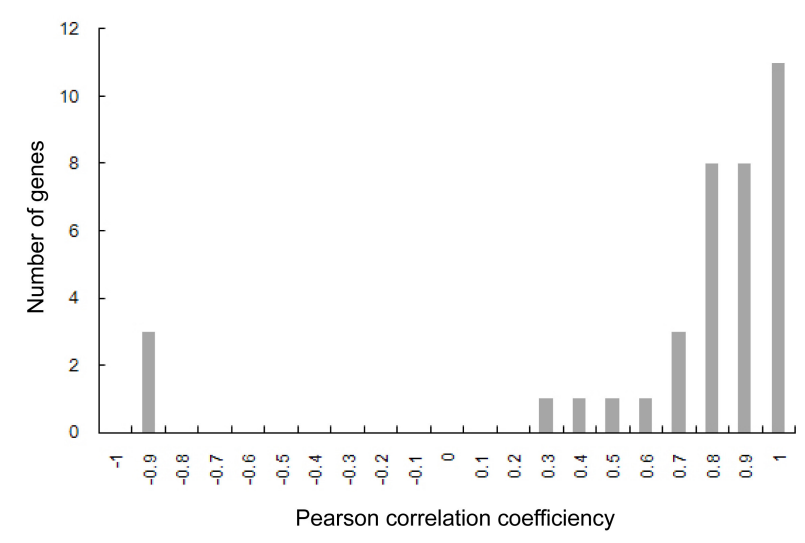

Fig. 2. Pearson correlation analysis of 37 proteins between the mRNA and protein level changes under rapamycin treatment. Changes in the mRNA and protein levels at the time point of 0,1 and $2 \mathrm{hr}$ after rapamycin treatment were compared and the consistency between them was determined by Pearson correlation analysis.

Table 1. Proteins that underwent protein abundance changes after rapamycin treatment for $1 \mathrm{hr}$

\begin{tabular}{ccc}
\hline Abundance change & Proteins \\
\hline Decreased & Tef1, Eno2, Tef2, Ssa2, Yef3, Tdh3, Rpl9b, Rpl8a, Rps0b, Rps21a, Rpl8b, Ykl071w, Met6, Rpl9a, Rpl16A, Psa1, \\
& Pgk1, Rps8b, Pma1, Tdh2, Spe2, Tkl1, Rpl2a, Rpa12, Rpl26b, Stm1, Rpc82, Sro9, Ygr250c, Sec61, Ths1, \\
& Trm112, Dhr2, Rps9a, Rpl23b, Egd2, Cwp2, Hal9, Hta2, Imd2, Zuo1, Hda3, Ppx1, Rps24b, Yor059c, Rpl5, Csm1, \\
& Mcm2, Htb2, Gln1, Scp160, Ctr2, Rpl35b, Bdf1, Cic1, Mon2, Sif2, Rpl41b, Lys1, Leu1, Rpl27b, Sqs1, Imd3, \\
& Vip1, Ssb2, Erg1, Xrs2, Ydr089w, Bop3, Ynl247w, Gnp1, Rps27a, Utp5, Ypr045c, Aat1, Yll054c, Shm2, Cin4, \\
& Suv3, Nsr1, Ils1, Bfr1, Arp6 \\
Increased & Yjl055w, Ahp1, Bik1, Pre6, Rck2, Ach1, Gdb1, Pbi2, Gad1, Rtc3, Mnn5, Spt6, Sok2, Mvd1, Eno1, Ald4, Rrp15, \\
& Mep2, Dig1, Hor2, Emc6, Hsp12, Add66, Ent4, Sum1, Fmp16, Yor289w, Opi10, Ypr090w, Dsf2, Mak10, Tdh1 \\
\hline
\end{tabular}


Table 2. Functional categorization of 37 proteins on the basis of $\mathrm{GO}$ biological process

\begin{tabular}{lcr}
\hline \multicolumn{1}{c}{ GO biological process } & \multicolumn{1}{c}{ Proteins } & $p-V a l u e$ \\
\hline Macromolecule biosynthetic process & Ils1, Rpl35b, Rpl27b, Rpl8b, Rps8b, Rpl23b, Rpl2a, Rpl9a, Rpl26b, Eno1, & $1.57 \mathrm{E}-08$ \\
& Rps24b, Rpl16a, Rps27a, Rpl8b, Rps0b, Yef3, Rpl9b, Ssb2, Rps9a, Rpl5 & \\
Translation & Ils1, Rpl35b, Rpl27b, Rpl8b, Rps8b, Rpl23b, Rpl2a, Rpl9a, Rpl26b, Rps24b, & $1.76 \mathrm{E}-08$ \\
& Rpl16a, Rps27a, Rpl8b, Rps0b, Yef3, Rpl9b, Ssb2, Rps9a, Rpl5 & \\
Regulation of vacuole fusion, non-autophagic & Eno1, Eno2 & $5.14 \mathrm{E}-03$ \\
GTP biosynthetic process & Imd2, Imd3 & $5.14 \mathrm{E}-03$ \\
\hline
\end{tabular}

busch, 2003) and also plays critical roles in expression of ribosomal protein genes (Powers and Walter, 1999). Therefore, the enrichment in the GO categories related with protein synthesis is consistent with the previous reports.

Notably, rapamycin treatment inhibited guanosine-5'triphosphate (GTP) biosynthetic process by decreasing the protein and mRNA level of Imd2 and Imd3. Imd2 and Imd3 are important enzymes for de novo GTP synthesis whose abundance and activity are correlated with cellular growth rate (Jackson et al., 1975). It is likely that repression of de novo GTP synthesis caused by decrease of Imd2 and Imd3 under rapamycin treatment may consequently contribute to inhibition of cell growth. This is consistent with the critical roles of TOR pathway in eukaryotic cell growth regulation.

\section{Discussion}

A number of regulatory mechanisms are involved in production of a protein. The expression level of mRNA is an important clue to understand cellular processes and functions. However, because of post-transcriptional mechanisms controlling the translation rate (McCarthy, 1998) and differential half-lives of mRNAs (Varshavsky, 1996), mRNA level alone does not reflect biological events precisely. Therefore, a quantitative proteomic analysis profiling the whole protein expressions is very useful for determination of cellular processes and functions.

In the present study, we systematically analyzed protein abundance changes using the yeast GFP-tagged collection under inhibition of TOR signaling by rapamycin treatment and showed that the proteins engaged in protein synthesis are the major regulatory targets of TOR pathway. Especially, 16 ribosomal proteins are included in the target list. This result is consistent with the previous reports. However, although 16 ribosomal proteins showed an abrupt decrease in protein abundance by rapamycin treatment, some other ribosomal proteins with long protein half-lives, such as Rpl43a and Rps19a (Belle et al., 2006), did not show any alteration in protein level under rapamycin treatment (data not shown). It is intriguing that abundance of some ribosomal proteins is immediately influenced by TOR inhibition while that of other ribosomal proteins does not have any relationship with TOR pathway. Further studies on this difference among ribosomal proteins will be helpful in elucidating the regulatory mechanism of TOR signaling pathway involved in translational control.

It is worthy to note that abundance of Imd2 and Imd3, the enzymes for de novo GTP synthesis, is decreased under inhibition of TORC1. GTP is essential to signal transduction and is converted to GDP through the action of GTPases. Recently, it has been reported that yeast Rag GTPases, Gtr1 and Gtr2, act as upstream regulators of TORC1 and play critical roles in coupling amino acid signal to TORC1 (Binda et al., 2009; Kim et al., 2008; Sancak et al., 2008). The fact that TORC1 is involved in GTP biosynthesis by regulating the protein level of Imd2 and Imd3 suggests that TOR pathway may affect the activity of several GTP-binding proteins including Gtr1 and Gtr2. It will be interesting to investigate if TOR pathway is really involved in regulation of the activity of GTP-binding proteins.

Previously, genome-wide functional profiling using the yeast deletion library has identified 396 mutant strains showing altered sensitivity to rapamycin (Xie et al., 2005). We compared this functional genomic result with our proteomic result. Among the 37 genes that show significant changes in the mRNA and protein level under rapamycin treatment, only two genes, PBI2 and MAK10, show alteration in rapamycin sensitivity upon deletion. This inconsistency between the genomic and proteomic responses to rapamycin is presumed to be due to differences in the experimental approaches for monitoring rapamycin effects, and is also indicative of a possibility that TOR signaling pathway influences cell physiology at numerous levels from genes to proteins. Thus, understanding the difference between the genomic and proteomic responses to rapamycin will give further insights into the regulatory mechanisms of TOR signaling pathway. 


\section{Acknowledgments}

This work was supported by grants from the $21 \mathrm{C}$ Frontier Functional Proteomics Project (FPR08A1-060) and the $21 \mathrm{C}$ Frontier Microbial Genomics and Application Center Program (MG-11-2008-09-004-00), Republic of Korea. C.S.S. and Y.J.C were supported by the BK21 Research Fellowship from the Ministry of Education, Science and Technology, Republic of Korea.

\section{References}

Ashburner, M., Ball, C.A., Blake, J.A., Botstein, D., Butler, H., Cherry, J.M., Davis, A.P., Dolinski, K., Dwight, S.S., Eppig, J.T., Harris, M.A., Hill, D.P., Issel-Tarver, L., Kasarskis, A., Lewis, S., Matese, J.C., Richardson, J.E., Ringwald, M., Rubin, G.M., and Sherlock, G. (2000). Gene ontology: tool for the unification of biology. The Gene Ontology Consortium. Nat. Genet. 25, 25-29.

Belle, A., Tanay, A., Bitincka, L., Shamir, R., and O'Shea, E.K. (2006). Quantification of protein half-lives in the budding yeast proteome. Proc. Natl. Acad. Sci. USA 103, 13004-13009.

Binda, M., Peli-Gulli, M.P., Bonfils, G., Panchaud, N., Urban, J., Sturgill, T.W., Loewith, R., and De Virgilio, C. (2009). The Vam6 GEF controls TORC1 by activating the EGO complex. Mol. Cell 35, 563-573.

Cherkasova, V.A., and Hinnebusch, A.G. (2003). Translational control by TOR and TAP42 through dephosphorylation of elF2alpha kinase GCN2. Genes Dev. 17, 859872.

Crick, F.H. (1958). On protein synthesis. Symp. Soc. Exp. Biol. 12, 138-163.

Hardwick, J.S., Kuruvilla, F.G., Tong, J.K., Shamji, A.F., and Schreiber, S.L. (1999). Rapamycin-modulated transcription defines the subset of nutrient-sensitive signaling pathways directly controlled by the Tor proteins. Proc. Natl. Acad. Sci. USA 96, 14866-14870.

Huh, W.K., Falvo, J.V., Gerke, L.C., Carroll, A.S., Howson, R.W., Weissman, J.S., and O'Shea, E.K. (2003). Global analysis of protein localization in budding yeast. Nature $425,686-691$.

Jackson, R.C., Weber, G., and Morris, H.P. (1975). IMP dehydrogenase, an enzyme linked with proliferation and malignancy. Nature 256, 331-333.

Jia, K., Chen, D., and Riddle, D.L. (2004). The TOR pathway interacts with the insulin signaling pathway to regulate $\mathrm{C}$. elegans larval development, metabolism and life span. Development 131, 3897-3906.

Kim, E., Goraksha-Hicks, P., Li, L., Neufeld, T.P., and Guan, K.L. (2008). Regulation of TORC1 by Rag GTPases in nutrient response. Nat. Cell. Biol. 10, 935-945.

Lee, M.W., Kim, B.J., Choi, H.K., Ryu, M.J., Kim, S.B.,
Kang, K.M., Cho, E.J., Youn, H.D., Huh, W.K., and Kim, S.T. (2007). Global protein expression profiling of budding yeast in response to DNA damage. Yeast 24, 145-154.

Loewith, R., Jacinto, E., Wullschleger, S., Lorberg, A., Crespo, J.L., Bonenfant, D., Oppliger, W., Jenoe, P., and Hall, M.N. (2002). Two TOR complexes, only one of which is rapamycin sensitive, have distinct roles in cell growth control. Mol. Cell. 10, 457-468.

Mayer, C., and Grummt, I. (2006). Ribosome biogenesis and cell growth: mTOR coordinates transcription by all three classes of nuclear RNA polymerases. Oncogene 25, 6384-6391.

McCarthy, J.E. (1998). Posttranscriptional control of gene expression in yeast. Microbiol. Mol. Biol. Rev. 62, 14921553.

Newman, J.R., Ghaemmaghami, S., Ihmels, J., Breslow, D.K., Noble, M., DeRisi, J.L., and Weissman, J.S. (2006). Single-cell proteomic analysis of $S$. cerevisiae reveals the architecture of biological noise. Nature 441, 840-846.

Powers, R.W. 3rd, Kaeberlein, M., Caldwell, S.D., Kennedy, B.K., and Fields, S. (2006). Extension of chronological life span in yeast by decreased TOR pathway signaling. Genes Dev. 20, 174-184.

Powers, T., and Walter, P. (1999). Regulation of ribosome biogenesis by the rapamycin-sensitive TOR-signaling pathway in Saccharomyces cerevisiae. Mol. Biol. Cell. 10, 987-1000.

Rohde, J., Heitman, J., and Cardenas, M.E. (2001). The TOR kinases link nutrient sensing to cell growth. J. Biol. Chem. 276, 9583-9586.

Sancak, Y., Peterson, T.R., Shaul, Y.D., Lindquist, R.A., Thoreen, C.C., Bar-Peled, L., and Sabatini, D.M. (2008). The Rag GTPases bind raptor and mediate amino acid signaling to mTORC1. Science 320, 1496-1501.

Sherman, F. (2002). Getting started with yeast. Methods Enzymol. 350, 3-41.

Song, Y.B., Jhun, M.A., Park, T., and Huh, W.K. (2009). Quantitative proteomic analysis of ribosomal protein L35b mutant of Saccharomyces cerevisiae. Biochim Biophys Acta.

Varshavsky, A. (1996). The N-end rule: functions, mysteries, uses. Proc. Natl. Acad. Sci. USA 93, 12142-12149.

Wullschleger, S., Loewith, R., and Hall, M.N. (2006). TOR signaling in growth and metabolism. Cell 124, 471-484.

Xie, M.W., Jin, F., Hwang, H., Hwang, S., Anand, V., Duncan, M.C., and Huang, J. (2005). Insights into TOR function and rapamycin response: chemical genomic profiling by using a high-density cell array method. Proc. Natl. Acad. Sci. USA 102, 7215-7220.

Zid, B.M., Rogers, A.N., Katewa, S.D., Vargas, M.A., Kolipinski, M.C., Lu, T.A., Benzer, S., and Kapahi, P. (2009). 4E-BP extends lifespan upon dietary restriction by enhancing mitochondrial activity in Drosophila. Cell 139, 149-160. 